\title{
AN OPTICAL IMAGING SEARCH FOR GRAVITATIONAL LENSES
}

\author{
S. Djorgovski \\ Palomar Observatory, California Institute of Technology \\ Pasadena, CA 91125, USA. \\ G. Meylan \\ European Southern Observatory \\ D-8046 Garching bei München, FRG.
}

\begin{abstract}
We are conducting an optical imaging search for gravitational lenses. A sample of known quasars was selected on the basis of high redshifts and apparently large absolute luminosities; these selection criteria significantly enhance the probability that a given QSO is gravitationally magnified. Our search has yielded so far at least one lensed QSO, and several promissing candidates.
\end{abstract}

From the extensive modeling of Turner, Ostriker \& Gott (1984), Ostriker \& Vietri (1986), and Blandford \& Kochanek (1987), we know that the distribution of lensed image separations should peak near 0.5 arcsec, so that the frequency of lensed QSO's increases rapidly with the resolution limit. This is even more pronounced in a flux-limited sample (= virtually all QSO samples), because of the magnification biasing. It is thus profitable to preselect the QSO's for a lens search by their apparent absolute luminosity: since the luminosity function falls off steeply at every redshift, an apparently high-luminosity QSO has a higher a priori probability of being lensed. One can improve the chances even further by selecting the highredshift QSO's (they have a large intercept length). A QSO sample preselected in this way should have it a considerably higher lens frequency than a "random" QSO catalog. The exact probability enhancement is hard to compute, since the biasing function depends on many unknowns (luminosity function of QSO's at different redshifts, mass distribution and clustering on large scales, cosmology, etc.).

Most QSO's are identified on a Schmidt plate material (e.g., the sky surveys), and thus with the resolution limit $\sim 2-5$ arcsec, depending on the seeing, magnitude contrast, etc. Thus, good-seeing CCD imaging of a well-selected sample of QSO's previously detected on a Schmidt plate material may right away produce some good lens candidates, with $\sim 1$ arcsec or larger separations. Discrepant colors can be used to eliminate spurious associations with foreground stars. Using a variety of image processing methods, one can push the detection of lensed pairs or multiplets well into the seeing limit. Any promissing candidates are then checked spectroscopically.

We have thus conducted for the last few years an optical (CCD) imaging search for lensed quasars from several different observatories. A sample of high- $z$ 
QSO's with apparently high absolute luminosities has been selected from the Hewitt \& Burbidge (1987) catalog. Most data were obtained as a byproduct of other projects: all of our "blank sky" frames (needed for CCD calibration) always have a carefully chosen QSO in them. So far, we have one very good lens candidate, UM 425 , described in the companion paper by Meylan \& Djorgovski, one probable binary quasar, PKS 1145-071 (Djorgovski et al. 1987), and several other promissing candidates. In addition, there are several cases of QSO's with foreground galaxies within a few arcsec, which should be useful for absorption-line studies. Our results will be described in more detail in future papers. An equivalent, very successful survey has been conducted independently by the Liège group (Surdej et al. 1987; Magain et al. 1988).

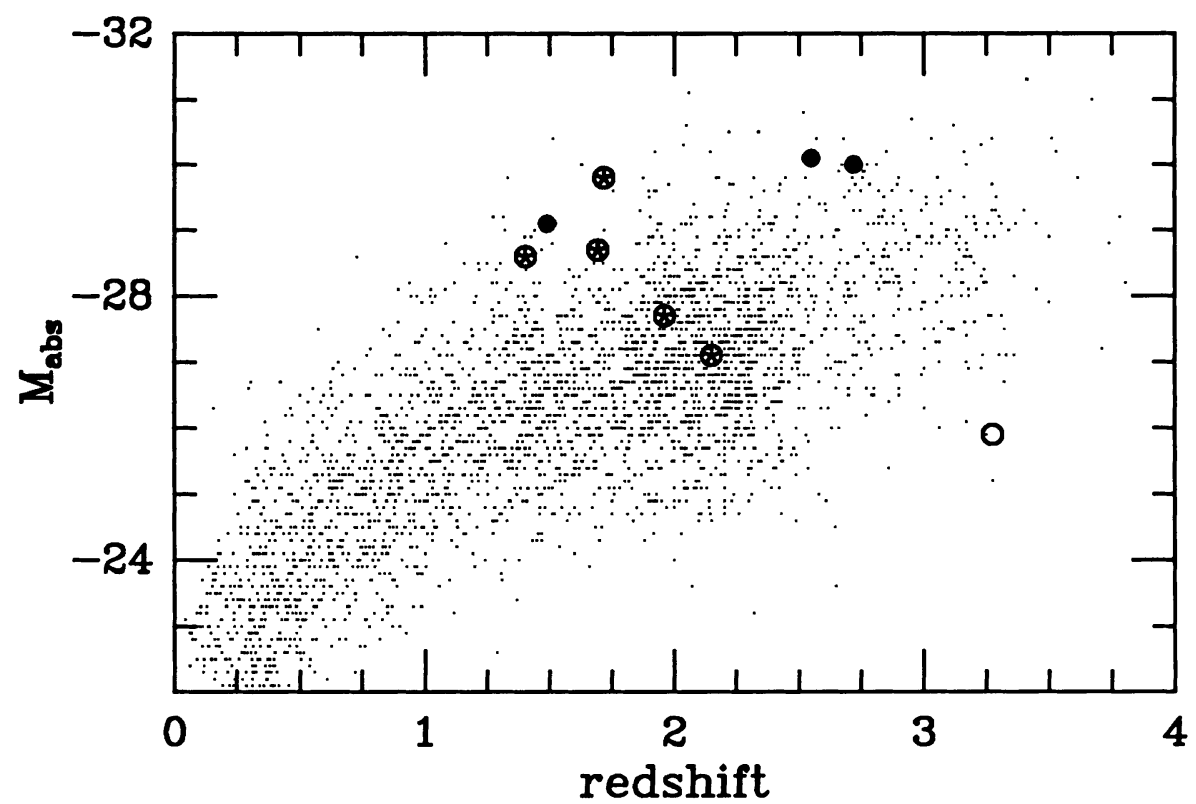

The bivariate distribution of redshifts and absolute magnitudes of QSO's from Hewitt \& Burbidge (1987). The solid circles denote the optically selected lenses, as described above; the star-crossed circles denote the optically selected, but serendipitously discovered lenses; and the open circle the one radio selected lens (other radio selected lenses have no measured redshifts as yet, but are also very faint). The effects of magnification biasing for the optically selected lenses are apparent.

\section{REFERENCES:}

Blandford, R., \& Kochanek, C. 1987, Ap.J. 321, 658.

Djorgovski, S., Perley, R., Meylan, G., \& McCarthy, P. 1987, Ap.J. 321, L1.

Hewitt, A., \& Burbidge, G. 1987, Ap.J.Suppl.Ser. 63, 1.

Magain, P. et al. 1988, Nature 334, 325.

Ostriker, J., \& Vietri, M. 1986, Ap.J. 300, 68.

Surdej, J. et al. 1987, Nature 329, 695.

Turner, E., Ostriker, J., \& Gott, J.R. 1984, Ap.J. 284, 1. 